\title{
BIOPHARMA IN PIEDMONT, ITALY
}

\author{
New career opportunities are emerging in the biopharmaceutical industry in Piedmont, \\ but there are challenges to realizing them, such as the need for internationalization.
}

Piedmont, in northern Italy, is located in a region of ancient and modern crossroads between Switzerland, France and Italy. Traditionally, the industrial strengths of the area have included textiles and mechanics, but owing to recent pressure on these industries, diversification into more knowledge-based sectors, including biopharmaceuticals, is being actively encouraged. The local presence and influence of several subsidiaries of global life-science companies, such as Serono, Bracco and Antibioticos, are important for this process, as is the highly productive nature of basic life-science research in Piedmont - the average ISI impact factor of the scientific publications from institutions in the region is high (5.7) ${ }^{1}$, which is comparable with Stockholm and Uppsala in Sweden, for example. There are several other factors that will be important in establishing Piedmont as a hub for biopharmaceutical research, which are discussed here, in addition to new initiatives aimed at enhancing the region's potential.

\section{Current climate}

A recent report found that the biotech clusters around Turin are the most cost-effective in Europe ${ }^{2}$. At present, there are $\sim 25$ biopharma companies of various sizes in Piedmont, employing a total of $\sim 1,500$ people. More than $50 \%$ of these companies have less than 10 employees.

The majority of the companies are located in the areas of Turin and Novara, in particular on two science parks: the BioIndustryPark of Canavese (BIPCa), and the Environment Park, Turin. There are close interactions between the biopharma companies and the major academic centres in the region, such as the University of Turin, the Polytechnical Institute of Turin and the Institute for Cancer Research (IRCC) Candiolo, but less so between the biopharma companies and the local subsidiaries of the global life-science companies, such as Serono. Moreover, there are too few interactions or collaborations with partners outside Italy.

\section{Exploiting the potential}

The need to establish international contacts and collaborations for biopharma research and development in Piedmont is acute. At present, hiring of non-Italian scientists/experts is rare, and more international scientific expertise on management teams and boards could considerably increase networking, collaborations and funding from abroad. As an example, the Piedmont biotech participation on European Union-funded grants is among the lowest of the Italian regions ${ }^{1}$. International venture capital is warranted. Increasing the internationalization of Italian biopharma companies could also encourage the return of Italian scientists working abroad, who could play a key role in establishing and developing the necessary links with foreign biopharma organizations.
Increasing the number of successful patent applications, which are of course pivotal for evolving biopharma companies, is another important and pressing matter. Only 21 biotechnology patents were granted in 2003 in Piedmont ${ }^{1}$, and the majority of this patent portfolio originated from Novara. This is too modest, particularly when compared with the excellent publication record of the region. Another apparent weakness is poor technology transfer within Piedmont, both to other regions of Italy and abroad. These issues deserve to be top priorities for local management teams and boards.

Nevertheless, a number of measures have already been launched to counteract the apparent weaknesses of the Piedmont biopharma industry ${ }^{3}$ (BOX 1). BIPCa, which houses 180 people, including start-ups and established biopharma companies, is particularly involved in these initiatives. Progress along these lines, bearing in mind in particular the need for internationalization, will be crucial in promoting and creating new career tracks for further development of science and industry in the region.

1. Calderini, M. et al. Measuring Piedmont's innovation performance in the biotechnology industry. Piemonte Biosciences Working Paper No. 9 (Oct 2004).

2. KPMG's Guide to International Business Costs [online],

$<$ http://www.competitivealternatives.com> (2004)

3. Piemonte Biosciences [online], <http://www. piemontelifesciences.com>

Acknowledgement

I would like to thank F. Conicella for stimulating discussions.

\section{Box 1 | Biopharma initiatives in Piedmont}

- The Piedmont Biosciences Initiative was founded by regional authorities in 2001. It is managed by the BioIndustryPark of Canavese (BIPCa), and its goals are to analyse life-science assets in the region.

- Eporgen, a private initiative, was created by local investors in November 2004. Eporgen is a seed-capital organization for start-up companies, and is nonexclusively linked to the 'Discovery' biopharma incubator at BIPCa (see below).

- Technology Scouting in Academia (TESINA) and Life Science Technology Network (LISTEN) were realized by funds from EU and the region. Their focus is on identification of major scientific breakthroughs and transfer of technologies.

- 'Discovery' is the new biopharma incubator of BIPCa, and functions as follows: selection of projects to be supported $\rightarrow$ pre-start-up support $\rightarrow$ Eporgen financial support for start-up $\rightarrow$ start-up assistance $\rightarrow$ development assistance $\rightarrow$ support by Eporgen to find new venture capital support.

- Eurobiocluster is a regional biopharma cluster that includes major research sites in France, Spain, Germany, Switzerland, Lombardy and Piedmont. This alliance was created in April 2005, and BIPCa functions as its management operator for the Piedmont region. 